\title{
Terremotos, solidaridad y movilización nacional
}

\author{
LUISA SCHONHAUT B. ${ }^{1}$
}

1. Profesor de Pediatría, Departamento de Pediatría. Facultad de Medicina Clínica Alemana-Universidad del Desarrollo.

\begin{abstract}
Earthquakes, solidarity and national mobilization

Introduction: Historically Chile has concentrated many of the largest earthquakes that have occurred worldwide, with great physical, psychological and economic impact on the population. Unlike previous events, the last earthquake /tsunami of February 27, 2010 has received major scientific attention. This article, insert in the commemorative issue of the Chilean Journal of Pediatrics, aims to give a historical overview of how earthquakes have positively shaped public policy and national identity. A historical analysis of the natural disasters in Chile between 1939 and 1960 is presented. Conclusions: The process of how coping with disasters has adapted to the prevailing socio-epidemiological reality, supporting high-impact public policies, is emphasized. In this context, child mental and emotional health has increasingly gained priority. The challenge is to develop, organize and implement strategies that respond to the real needs of the affected population.
\end{abstract}

(Key words: Disasters, earthquake, pediatrics history).

Rev Chil Pediatr 2013; 84 (1): 20-25

\section{RESUMEN}

Introducción: Históricamente el territorio de Chile ha concentrando varios de los movimientos telúricos de mayor magnitud que se han registrado a nivel mundial, con gran impacto físico, psicológico y económico en la población. A diferencia de eventos anteriores, el último terremoto/maremoto, del 27 de febrero de 2010 ha sido registrado en una importante producción científica. El presente artículo, inserto en el número conmemorativo de la revista Chilena de Pediatría, tiene por objetivo dar una mirada histórica de cómo los terremotos han logrado moldear positivamente las políticas públicas e identidad nacional. Para ello se realiza un análisis histórico de los desastres naturales ocurridos en Chile los años 1939 y 1960. Conclusiones: Se destaca como el enfrentamiento de desastres se ha ido adecuando a la realidad socio-epidemiológica predominante, sustentando políticas públicas de alto impacto. En este marco, la salud mental y emocional de los niños ha ido adquiriendo cada vez mayor prioridad. El desafío es desarrollar, organizar e implementar estrategias que respondan a las reales necesidades de la población afectada.

(Palabras clave: Desastres, terremoto, historia pediatría).

Rev Chil Pediatr 2013; 84 (1): 20-25

Recibido el 09 de noviembre de 2012, devuelto para corregir el 03 de diciembre de 2012, segunda versión el 19 de diciembre de 2012, aceptado para publicación el 14 de enero de 2013.

Este trabajo cumple con los requisitos sobre consentimiento /asentimiento informado, comité de ética, financiamiento, estudios animales y sobre la ausencia de conflictos de intereses según corresponda.

Correspondencia a:

Dra. Luisa Schonhaut Berman

E-mail: Ischonhaut@alemana.cl 


\section{Introducción}

Según definición de la Organización Mundial de la Salud un desastre es "una situación de ruptura del funcionamiento normal de un sistema o comunidad, cuyos efectos en las personas, así como las pérdidas y daños materiales o ambientales, sobrepasan la capacidad de esa sociedad o comunidad para responder y recuperarse de la situación". Es decir, el grado de daño es tal, que hace necesario movilizar recursos desde distintos sectores con el fin de suplir las necesidades básicas para la sobrevivencia y rescate de la población afectada.

Debido a la naturaleza telúrica del territorio, Chile ha concentrando varios de los movimientos telúricos de mayor magnitud que se han registrado a nivel mundial. De acuerdo con la información del Servicio Sismológico del Departamento de Geofísica de la Universidad de Chile, durante el siglo XX e inicios del XXI fueron registrados 75 sismos que podrían ser catalogados como terremotos. Los más emblemáticos fueron los de 1906 (Valparaíso), 1939 (Chillán), 1960 (Valdivia), 1985 (San Antonio), y el reciente de 2010 (Cobquecura-Concepción) $)^{2}$.

Cuando repasamos nuestra historia, podemos ver cuánto hemos aprendido y adecuado el enfrentamiento del desastre a la realidad socio-epidemiológica predominante, sustentando políticas públicas de alto impacto.

No obstante, al enfrentar cada nuevo evento, quedamos con una amarga sensación de deficiencia en la organización e improvisación ${ }^{3}$. Probablemente esta mirada tan crítica se debe a que "las sociedades civilizadas aceptan cada vez menos los daños que pueden ser evitados o eventuales impactos en la salud que pueden ser minimizados" (Serra I. post terremoto 1985) $)^{4}$. Cincuenta años atrás, el Dr. Alfredo Avendaño, quien participó como interno en la atención de damnificados por el terremoto de 1960, relató en un discurso muy crítico su experiencia que "puede volverse valiosa, al juntarse y compararse con muchas otras, para dar base a un Programa de Salud Pública"5.

El presente artículo tiene por objeto dar una mirada histórica de cómo los terremotos han logrado moldear no sólo la geografía, sino también las políticas públicas e identidad nacional.

\section{La solidaridad nacional tras el terremoto de 1939}

En los años 30 Chile venía saliendo de una gran crisis económica, la mortalidad materno infantil era una de las más altas del mundo, estimándose en 225 a 236 fallecidos por 1.000 recién nacidos vivos. "las enfermedades más frecuentes que atacan al organismo infantil son las causadas por los defectos en la alimentación, por la infección y la falta de higiene en general.". Gran parte de la población crecía en un ambiente de miseria, "canción que mece al recién nacido y al lactante. Ella continúa al compás del mismo sonsonete flagelando al preescolar y al escolar de un gran número de hogares chilenos... a este ambiente, diríamos casero, cabe añadir el panorama sanitario exterior-tampoco muy bello por cierto-, que agudiza los problemas descritos, al encontrarnos ante serias deficiencias en el suministro de agua potable, así como en los servicios de alcantarillado, recogida de basuras, etc." (Pascual P. 1947) ${ }^{6}$.

En Santiago había apenas 2 hospitales pediátricos, donde los recursos humanos y arsenal terapéutico escaseaban ${ }^{7}$. Pocos artículos relatan las condiciones en aquella época en la provincias, donde la situación era aún más compleja "la mortalidad de la madre es enorme en los departamentos de las distintas provincias y esto hay que corregirlo a breve plazo si no queremos el despueble de todos ellos". (Guzmán L. 1939) ${ }^{8}$.

Ese era el escenario, cuando el país fue sacudido por uno de los terremotos más devastadores conocidos en la historia, la noche del 24 de enero de 1939, dejando un saldo de casi 30.000 muertos y decenas de miles de heridos y damnificados. En palabras del médico $\mathrm{y}$ diputado por Concepción, Natalio Berman "En la histórica ciudad, cuna de O'Higgins, no han quedado más de cinco edificios en pie. El resto forma un hacinamiento de ruinas. Las calles presentan un aspecto desolado. Los que las recorren llevan impreso para siempre el sello que les dejó la tragedia. Caminan, sin 
saber para dónde, como empujados por una mano invisible y por un recuerdo que persiste con características trágicas" 9 .

Parece increíble cómo, a pesar que el panorama nacional ya era sombrío, la tragedia movilizó numerosos voluntarios y despertó una enorme solidaridad ciudadana. El Director General de Sanidad de aquellos años, Dr. Leonardo Guzmán, relataría “... Apenas me fue posible, me embarqué en Valparaíso en el vapor 'Teno' con rumbo al puerto de Talcahuano, haciéndome acompañar por 16 médicos, 15 estudiantes de medicina, 14 practicantes $y$ 12 camilleros..."8.

Entre las estrategias de acción fundamentales se definió: a) trasladar los enfermos graves a ciudades no afectas por el sismo; b) dar albergue a los damnificados y encontrar asilo permanente a los miles de huérfanos; c) implementar una estrecha vigilancia epidemiológica y d) establecer medidas profilácticas como campañas masivas de vacunación, saneamiento ambiental y cloración de las aguas.

Documentos históricos refieren que el terremoto de 1939 marcó el inicio de las grandes campañas de ayuda solidaria ${ }^{10}$ y ayudó al fortalecimiento del rol estatal a nivel del desarro1lo, organización y coordinación de políticas sociales $^{11}$, proceso que se vio coronado una década más tarde, con el establecimiento del Servicio Nacional de Salud (SNS) ${ }^{12,13}$.

\section{Los huérfanos del Terremoto de 1960}

Tras casi una década de funcionamiento del SNS la tasa de natalidad era alta, prácticamente el doble de nuestros días, estimándose en 38,6 por 1.000 y la mortalidad infantil que aún era elevada, había descendido a $125-127$ por 1.000 nacidos vivos ${ }^{14}$. No obstante las cifras eran alentadoras, los terremotos y maremoto de los días 20 y 21 de mayo de 1960, pusieron en evidencia la profunda inequidad económica, cultural y doctrinaria, al sacudir una de las zonas más pobres del país ${ }^{5,15}$.

Uno de los sucesos que generó gran impacto a nivel nacional fue el sacrificio de un niño de 7 años como parte de una ceremonia religiosa de desagravio "guillantunes" de pobladores indígenas, que consideraban que el devastador terremoto era producto del enojo de los dio$\operatorname{ses}^{5,16}$. Afortunadamente, desde aquel trágico episodio, no se ha conocido nuevos sacrificios humanos en nuestro país.

Como ocurre en situaciones de desastre, el SNS por sí sólo no tenía la capacidad de dar respuesta a toda la desolación y miseria en que quedó sumida la zona Sur del país. Se recibió gran apoyo nacional e internacional para cumplir con la atención de emergencia, además de realizar labores de "inmunización, educación sanitaria e higiene mental'. ${ }^{17}$. Ejemplo de la solidaridad nacional, una vez más manifiesta frente al desastre natural, fue la campaña "un cigarrillo para el Sur", recopilando a pocos días de ocurrido el siniestro $121 \mathrm{~kg}$ de cigarrillos para las ciudades de Chiloé, Valdivia y Osorno ${ }^{18}$.

La extrema situación que se estaba viviendo provocó "manifestaciones y estados psicológicos peculiares" 16 , tanto en la población afectada como en los voluntarios, reportándose una actitud generalizada de apatía, pero "mínima" incidencia de cuadros psiquiátricos secundarios $^{19,17}$. Las "modificación psicológicas", sumadas a la insalubridad, hambruna y enfermedades infectocontagiosas que se propagaban en los albergues, además de los movimientos telúricos reiterados y la amenaza de desborde del lago Riñihue, generó tal clima de inseguridad que "provocó la salida, entre gente de recursos, de mujeres y niños, y lo que fue más grave, la evacuación masiva o indiscriminada de gran cantidad de niños humildes que fueron separados de sus madres" para acogerlos en familias o instituciones públicas. ..." $a l p a$ recer las madres, cuando se les describía las ventajas y buena situación que su niño iba a tener en el lugar a que fuera trasladado, accedian sin pensarlo mucho y sin hacer mayores averiguaciones sobre cuándo y cómo lo irían a recuperar" 17 .

Ciudades como Valparaíso, Santiago y Osorno recibían las caravanas de niños descalzos para "brindarles la hospitalidad amable y cariñosa que hará olvidar los trágicos dias que han vivido" ${ }^{18}$. En un análisis de 2030 niños que llegaron a la ciudad de Santiago, se confirmó la orfandad en el $0,3 \%$ de los casos, 
correspondiendo a 6 niños, todos ellos pertenecientes a una familia de Peulla. Destacaba que sólo $16(0,7 \%)$ presentaron problemas conductuales "provocados en su mayor parte por desadaptación al ambiente o alejamiento de sus familiares, indicándose en estos casos el traslado de estos niños a otras colocaciones ola la Casa Central del Consejo de Defensa del Niño, para su tratamiento"20.

Sin duda la intención era loable y al parecer habitual en las campañas de protección de los menores, como ocurriera en situaciones de guerra o epidemia, ejemplificado por los preventorios, que tuvieron su apogeo a mediados del siglo recién pasado ${ }^{21}$. A pesar de la aparente "normalidad" de la medida adaptada, algunos psiquiatras de la época consideraban ésta "una política desafortunada"17. Alberto Gallinato, por un lado, y Max Letelier junto a Francisco Barilari por otro, advertían sobre "los desastrosos efectos producidos al separar a niños pequeños del lado de sus madres" 17 con riesgo de "provocar neurosis y otros problemas sociales, a raíz de la desadaptación" ${ }^{22}$. Paradójicamente el año 1939, el Dr. Victor Grossi, entonces Jefe del Servicio de Salubridad de la ciudad de Concepción, estableció como política, mientras sea posible "no desvincular a los niños de su zona y de su ambiente"23.

La participación de psiquiatras y salubristas quedó rigurosamente documentada en las Sextas Jornadas de la Sociedad Chilena de Salubridad de 1960. Veinticinco años después, posterior al terremoto de 1985 , se describía la importancia de la intervención psicológica de la población afectada ya sea directa o indirectamente, para prevenir secuelas a largo plazo $^{24}$.

\section{Terremotos y epidemias}

La crisis originada en los desastres naturales, provocan incertidumbre, angustia y estrés entre otra mezcla de emociones, las que van tejiendo historias, creencias y mitos. Uno de los mitos más comunes está asociado a la emergencia y virulencia de epidemias infectocontagiosas. Con motivo del gran sismo de Lima de 1940 y la difteria, el Dr. Carlos Bazan publicaría en nuestra revista “...tenemos la impre- sión de que a raíz del terremoto se inició entre nosotros un brote epidémico de gran consideración y de manifiesta malignidad, brote que prosperó al amparo de la falta de vacunación intensiva." 25 .

El temor a las epidemias motiva la implementación de campañas masivas de vacunación, quizás más fluidas en estas situaciones por la enorme entrega de los afectados. Así por ejemplo, en relación al movimiento telúrico de 1939 se vacunó contra fiebre tifoidea a más de 200.000 personas, siendo esta "la más extensa vacunación de que haya recuerdo en la historia sanitaria del orbe entre la población civil"8.

No obstante, se ha demostrado que las epidemias de enfermedades transmisibles son infrecuentes después de un desastre natural, salvo entre las personas albergadas en condiciones insalubres ${ }^{26,27}$. Seguramente ese fuese el motivo que, luego del terremoto del 2010, se administró inmunización contra la influenza AH1N1 a un significativo número de niños de regiones afectadas y a todos los amparados en carpas, albergues, campamentos y aldeas, además de los voluntarios y funcionarios expuestos $^{28}$.

Si bien las vacunas son herramientas profilácticas de indiscutible costo-beneficio, no son eficaces frente a la otra índole de epidemias que surgen tras un desastre y son las relacionadas con los problemas de salud mental, que siendo esta una epidemia tan masiva, podría pasar desapercibida por la población tanto usuaria como prestadora de servicio, toda ella golpeada emocionalmente cuando no física y económicamente.

Tras el último gran terremoto, se documentaron elevados niveles de estrés postraumático tanto en los niños como en sus cuidadores ${ }^{29-31}$. Y si bien, rápidamente se instalaron servicios de apoyo psicológico, se comprobó una baja consulta espontánea debido a este tema, predominando las derivaciones relacionadas con patologías del orden disruptivo preexistente ${ }^{32}$. Quizás este fenómeno de apatía y pasividad sea explicado por las "modificación psicológicas" y potenciado por la sobreintervención, no siempre tan planificada y dirigida a las necesidades de la población, aspecto ya registrado en relación a los terremotos de 1939 y 1960. 
Pero no todo es negativo, de las experiencias difíciles y de los desastre también salen experiencias enriquecedoras, concepto nominado por la psicología positiva como resiliencia y crecimiento postraumático, respuestas que pueden explicar en parte el crecimiento que ha tenido el país tanto a nivel de confección de políticas públicas, movimientos de solidaridad e identidad nacional.

\section{Conclusiones}

Dada la naturaleza sísmica del territorio chileno es imprescindible seguir desarrollando políticas y programas de salud pública que nos ayuden a enfrentar nuevos eventos, los que tenemos la certeza que nos visitarán frecuentemente, aunque no sepamos exactamente cuándo ni dónde será el próximo.

Parece increíble cómo la preocupación por la salud mental y emocional de los niños ha ido ocupando cada vez un lugar más prioritario dentro de las actividades de rescate. No obstante, aún queda como tarea el desarrollo de estrategias de atención post desastre que sean implementadas desde un enfoque de derechos, a las necesidades de la población afectada ${ }^{33}$, evitando la desorganización y sobre-intervención.

Si bien este manuscrito se centra en los eventos de los años 1939 y 1960, los lectores podrán profundizar en el desastre ocurrido hace apenas tres años en el terremoto maremoto del 27 de febrero de 2010, registrado gracias a una importante producción científica, parte de ella publicada en este número conmemorativo de la revista Chilena de Pediatría.

\section{Referencias}

1.- Organización Panamericana de la Salud: Logística y Gestión de Suministros Humanitarios en el Sector Salud. Washington, D.C.: OPS/OMS; 2001; p. 1.

2.- Universidad de Chile: Departamento de Geofísica. Servicio Sismológico. "Sismos importantes y/o destructivos (1570-a la fecha). Magnitud Ms igual o mayor a 7.0". Disponible en http://ssn.dgf.uchile.cl/seismo.html (consultado 8/11/12).
3.- San Martín P: Desafíos post terremoto, alcances para una política pública frente a catástrofes naturales. http:// www.ignire.cl/columnas-de-opinion/desaf-os-postterremoto-alcances-para-una-pol-tica-p

4.- Serra I: Salud Pública y Desastres Naturales. Cuad, Méd. Soc 1986; 27 (1): 5-11.

5.- Avendaño A: Experiencia de los Internos de Medicina durante el sismo de mayo de 1960. Publicación Sextas Jornadas. Sociedad Chilena de Salubridad, Santiago SNS 1960

6.- Pascual P: aspectos medico-sociales del niño en chile. Rev Chil Pediatr 1947; 18 (5): 333-52 (citado 12/10/12) Disponible en: http://www.scielo. cl/scielo.php?script=sci_arttext\&pid=S0370-4106194 7000500003\&lng=es\&nrm=iso

7.- Baeza Goñi A, Schonhaut L: hospitalizacion del lactante. Rev Chil Pediatr 2012; 83 (3): 290-4.

8.- Guzmán L: Actuación de la Dirección General de Sanidad de Chile a raíz del terremoto de enero 1939. Boletín de la Oficina Sanitaria Panamericana (OSP) 1939; 18 (11): 1044-8.

9.- Berman N: Entrevista Zigzag en Chile en el siglo XX. VI-e Virtual Educativo / Chile en el siglo XX. (consultado 9/9/12). Disponible en http://chile-catastrofestragedias.blogspot.com/2010/07/terremoto-chillan-24de-enero-de-1939.html

10.- Memoria Chilena: http://www.memoriachilena.cl/ temas/dest.asp?id=terremotoschillan

11.- Rojas Flores J: Historia de la infancia en el Chile republicano, 1810-2010. World color, Chile 2010.

12.- Mardones-Restat F: Políticas de alimentación y nutrición, en los planes de salud en chile. Rev Chil nutr 2003; 30 ( suppl.1): 195-7.

13.- Montoya-Aguilar C: La atención primaria de salud en Chile y el papel de los médicos. Nuevos objetivos en el año 2010. Cuad Méd Soc (Chile) 2010; 50 (3): 167-77. (consultado 9/9/12). Disponible en http://www.colegiomedico.cl/Portals/0/files/biblioteca/publicaciones/ cuadernos $/ 50$ 3.pdf\#page $=5$.

14.- San Martin H, Merino R: El ambiente de vida del niño chileno. Rev Chil Pediatr 1960; 1957-72.

15.- Armijo R: Discurso de Apertura Sextas Jornadas salubridad 1960. Publicación Sextas Jornadas. Sociedad Chilena de Salubridad, Santiago SNS 1960.

16.- Muñoz L: Salud Mental en las zonas afectadas por los terremotos de mayo de 1960. Publicación Sextas Jornadas. Sociedad Chilena de Salubridad, Santiago SNS 1960.

17.- Letelier M, Barilari F: Observaciones psicológicas recogidas en las ciudades de Valdivia y Puerto Montt 
con ocasión de la catástrofe ocurrida en mayo de1960. Publicación Sextas Jornadas. Sociedad Chilena de Salubridad, Santiago SNS 1960.

18.- Diario La Prensa 11/06/1960, (consultado 10/11/12) Disponible en http://www.terremoto1960.cl/index. php?option=com_content\&view $=$ article \&id=296\&Ite mid=41\&lang=es

19.- Diario La Prensa 29/06/1960, (consultado 10/11/12) Disponible en http://www.terremoto1960.cl/index. php?option $=$ com_content $\&$ view $=$ article $\&$ id $=296 \&$ Ite $\mathrm{mid}=41$ \&lang $=\mathrm{es}$

20.- Infante R, López R, Alvear I, Volochinsky A: Informe sobre colocación de niños damnificados trasladados de la zona sur a la Provincia de Santiago. Rev Chil Pediatr 1961; 305-7.

21.- Schonhaut L: El Preventorio Infantil de Montaña de la Cruz Roja Chilena. Rev Chil Pediatr 2008; 79 (2): 184-190 http://www.scielo.cl/scielo.php?script=sci arttext\&pid=S0370-41062008000200011\&lng=es\&nr $\mathrm{m}=\mathrm{iso} \& \mathrm{t} \operatorname{lng}=\mathrm{es}$

22.- Gallinato A: Niños evacuados candidatos a neurosis, en Ercilla, Santiago, N 1309, 22/junio/1960, p. 9 Citado en Rojas Flores J: historia de la infancia en el Chile republicano, 1810-2010. World color, Chile 2010.

23.- Grossi V: Citado en publicación Sextas Jornadas. Sociedad Chilena de Salubridad, Santiago SNS 1960.

24.- http://cidbimena.desastres.hn/docum/crid/Nov-Dic2003/ pdf/spa/doc9134/doc9134-contenido.pdf

25.- Bazan C: Aspectos Clínicos de la Difteria en nuestro medio.(1941-1951) Rev Chil Pediatr 1952:508-516. (Consultado 2-10-12) Disponible en http://www.scielo. $\mathrm{cl} / \mathrm{pdf} / \mathrm{rcp} / \mathrm{v} 23 \mathrm{n} 11 / \mathrm{art} 07 . \mathrm{pdf}$

26.- Toole M: Enfermedades Transmisibles y su control en Noji E. Impacto de los desastres en la salud pública. (Consultado 2-10-12) Disponbile en: http://www.paho. org/spanish/ped/impacto.htm

27.- Ledermann $W$ : Terremotos y Microbiota. Rev Chil Pediatr 2010; 81 (2): 101-102.

28.- http://www.gobiernodechile.cl/media/2010/05/SALUD. pdf

29.- Cova F, Valdivia M, Rincón $P$, et al: Estrés postraumático en población infantojuvenil post 27F. Rev Chil Pediatr 2013; 84 (1): 32-41.

30.- Briceño AAM, Abufhele MM, Dávila DA, et al: Estrés postraumático en escolares a 8 meses del 27F. Rev Chil Pediatr 2013; 84 (1): 42-50.

31.- Bedregal GP, Camila Carvallo PC, Hernández LlV, Prado RP: Estrés post-traumático post terremoto $27 \mathrm{~F}$ en cuidadores de niños preescolares. Factores asociados del cuidador, de la familia y la crianza. Rev Chil Pediatr 2013; 84 (1): 51-58.

32.- Sommer AK, Abufhele MM, Briceño AAM, et al: Intervención de salud mental en niños expuestos a desastre natural. Rev Chil Pediatr 2013; 84 (1): 59-67.

33.- Arbour MC, Murray K, Arriet F, Moraga C, Cordero Vega M: Lessons from the Chilean Earthquake: How a Human Rights Framework Facilitates Disaster. Health and human rights 2011; 13 (1): E70-81. 